\title{
Comparative study between sub-coronal and peno-scrotal approach of semi-rigid penile prosthesis implantation
}

\author{
Basiony Abd Alhady, Mohamed Abdelshafy, Osama Al Shahat, Mourad Mahmoud, Ahmed \\ Taha, Abdel Rahman Awadeen
}

Department of Plastic and Reconstructive Surgery, Faculty of Medicine, Al-Azhar University

Corresponding author: Basiony Abd Alhady, email: nimdee030@gmail.com

\begin{abstract}
Aim of the work: study was done to compare the benefits and risks for patients doing insertion of penile prostheses for treatment of erectile dysfunction by two different approaches (sub-coronal and penoscrotal) and to try discover the most suitable one. Patients and Methods: a total of 24 patients were included in this study that had all complaining from sever erectile dysfunction. All patients were subjected to pre as well as post-operative fixed assessment. International Index of Erectile Function (IIEF), Erectile Dysfunction Inventory of Treatment Satisfaction (EDITS). Patients were classified into two groups according to the approach of implantation. Procedure was done for every individual patient. Analysis of the final results was done to compare the different procedures and evaluate them individually and comparatively in an effort to categorize the overall benefit of each procedure. Results: each of the two test groups showed a significant improvement and satisfaction of complaining, as well as a significant difference between the two groups as regard(EDITS) ( $p$-value $<0.005$ ). Conclusion: In general, management of erectile dysfunction had a strong impact on the quality of life of the patients especially who underwent the penile prostheses implantation, most significantly affecting their "selfesteem", the penoscrotal approach was more suitable for semi-rigid penile prostheses than subcoronal one.
\end{abstract}

\section{INTRODUCTION}

Erectile dysfunction (ED) is defined as the consistent inability to achieve and maintain a penile erection adequate for satisfactory sexual intercourse. ED may substantially decrease the quality of life for both the man and his partner (i). ED may be related to vascular disease, neurological disease, hormonal defects, cavernosal fibrosis from Peyronie's disease, ischaemic priapism or diabetes mellitus ${ }^{(2)}$. The first-line treatment for ED was the oral administration of a phosphodiesterase type $\mathrm{V}$ inhibitor. The phosphodiesterase type $\mathrm{V}$ inhibitor had revolutionized ED management due to the inhibitor's high rates of efficacy and favourable adverse-effects profile (3). In patients who did not respond to the medical management of $\mathrm{ED}$, penile prosthesis implantation (PPI) was a definitive option for ED management. Modern penile prostheses have been available since the early 197 os $^{(4)}$.

With regard to the surgical technique, various approaches had been described for implanting penile prostheses: the dorsal subcoronal, penile proximal, longitudinal penoscrotal, transverse penoscrotal perineal and combined incisions (5).

\section{AIM of the WORK}

The aim of this study was to compare the benefits and risks for patients doing insertion of penile prostheses for treatment of erectile dysfunction by two different approaches (subcoronal and penoscrotal) and to discover the most suitable one.

\section{PATIENTS and METHODS}

A total of 24 patients were included in this study that had all complaining from sever erectile dysfunction, they had been suffered for more than 1 year at least.The study was executed in the period from January 2016 to January 2018 in Al-Azhar University Hospital (Plastic Surgery and Urology Surgery Department). The study was approved by the Ethics Board of Al-Azhar University.

The mean age of the patients was 46 years. There were 9 cases heavy smokers, 15 patients had controlled diabetes and 9 patients had hyper lipidemia. All patients were within normal range of hormonal profile. All patients and their partners were subjected to pre as well as post-operative fixed assessment such as: International Index of Erectile 
Function(IIEF),Erectile Dysfunction Inventory of Treatment Satisfaction (EDITS).They were categorized randomly into two groups according to surgical approaches for semi rigid penile prosthesis implantation:

Group 1: penoscrotal approache, included 12 patients with erectile dysfunction.

Group 2: subcoronal approache, included 12 patients with erectile dysfunction.

The follow-up period for patients in this study ranged from 6 months to 1 year. The data were coded, entered and processed on computer by using SPSS (Version 18).The results were represented in tabular and diagrammatic forms then interpreted. Mean, standard deviation, range, frequency and percentage were used as descriptive statistics. The following tests were done: Chi-Square test $\mathrm{X}^{2}$ and Student's t-test was used. $\mathrm{P}$ value was considered significant as the following:

$* \mathrm{P}>0.05$ : Non significant $* \mathrm{P} \leq 0.05$ : Significant

\section{RESULTS}

A total of 24 patients were included in this study. They had all complaining from sever erectile dysfunction and they were suffered for more than 1 year at least. The mean age of the patients was 46 years. There were 9 cases heavy smokers, 15 patients had controlled diabetes and 9 patients had hyper lipidemia. All patients were within normal range of hormonal profile. All patients were subjected to pre as well as post-operative assessment by : International Index of Erectile Function (IIEF),Erectile Dysfunction Inventory of Treatment Satisfaction (EDITS).

Peno-scrotal group showed a significant satisfaction as regard (EDITS) ( $p$-value $<0.05)$. However, no significant difference was found in IIEF ( $p>0.05)$.In addition we not there are glans problem occurred mostly in sub coronal more than in penoscrotal one. The assessment was individual and photographs were used. Patient satisfaction was assessed by direct patient questioning and included subjective and objective feedback on overall satisfaction following penile prostheses implantation (Fig. 1).

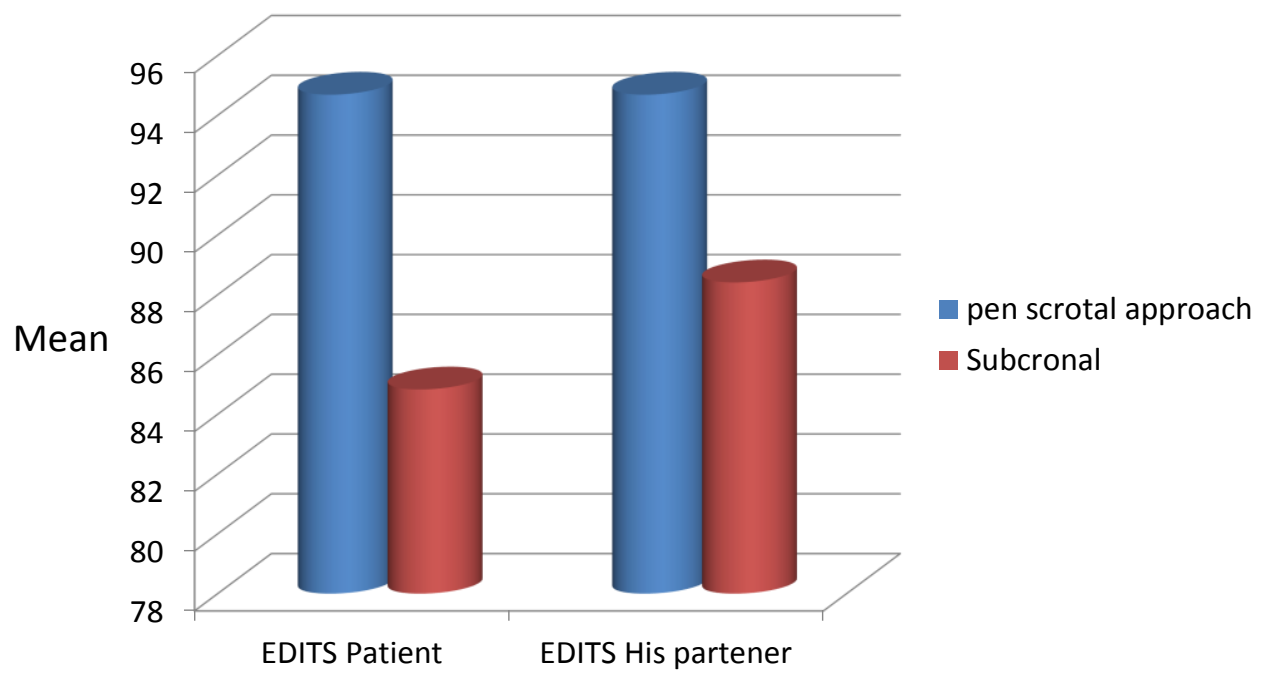

Figure 1: comparison between peno-scrotal approach and subcoronal regarding EDITS

The most common complications were crossover 4 cases $(1.7 \%)$ and wound dehisance 8 cases $(33.3 \%)$. in these patients repeated dressing under sterile precautions was performed; this complication was successfully managed in all patients. One case (4.2\%) had wound infections; he was treated by intra-venous antibiotics and wound drainage. 

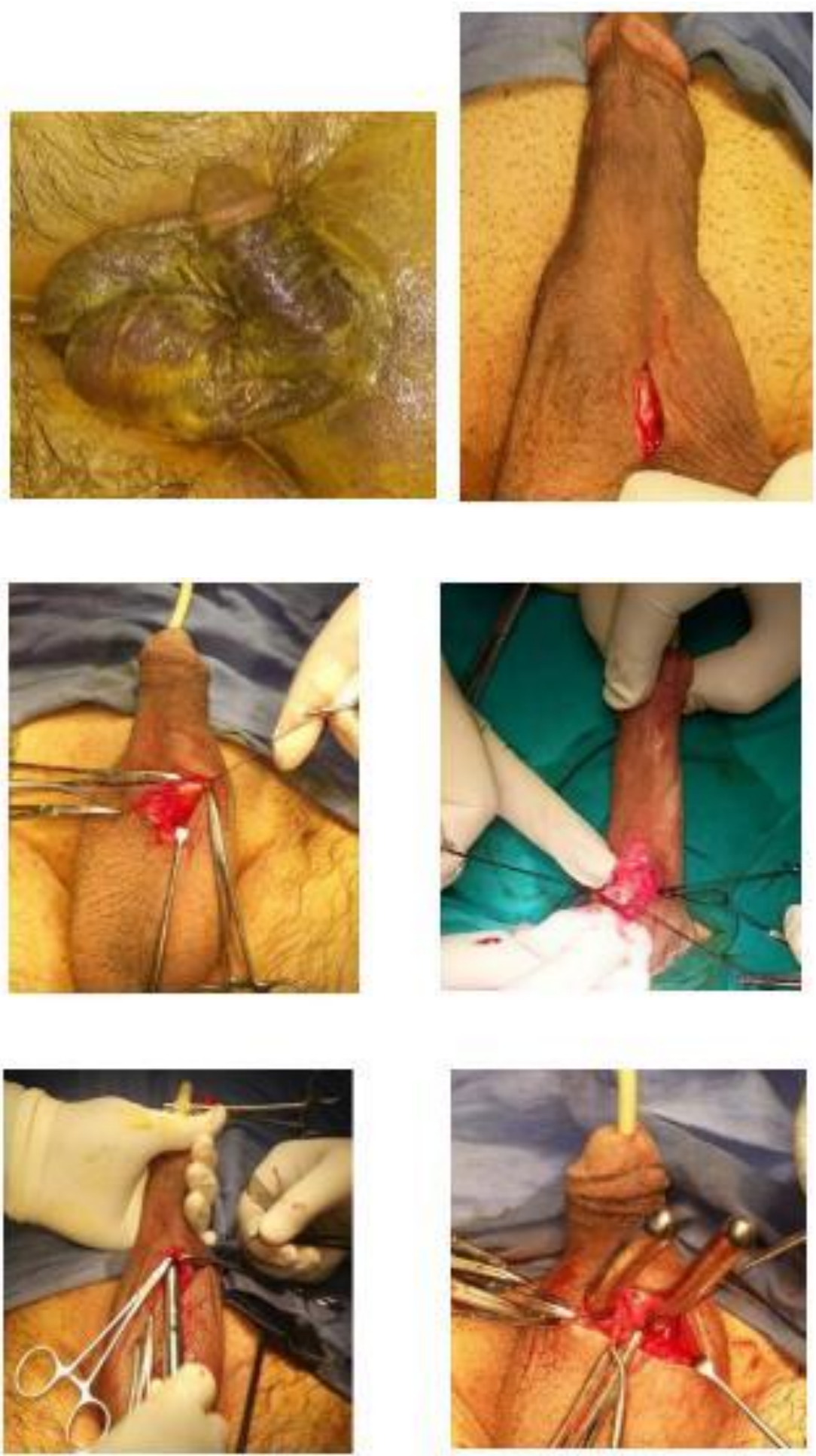

Figure 2: steps of penile prostheses implantation by penoscrotal approach 

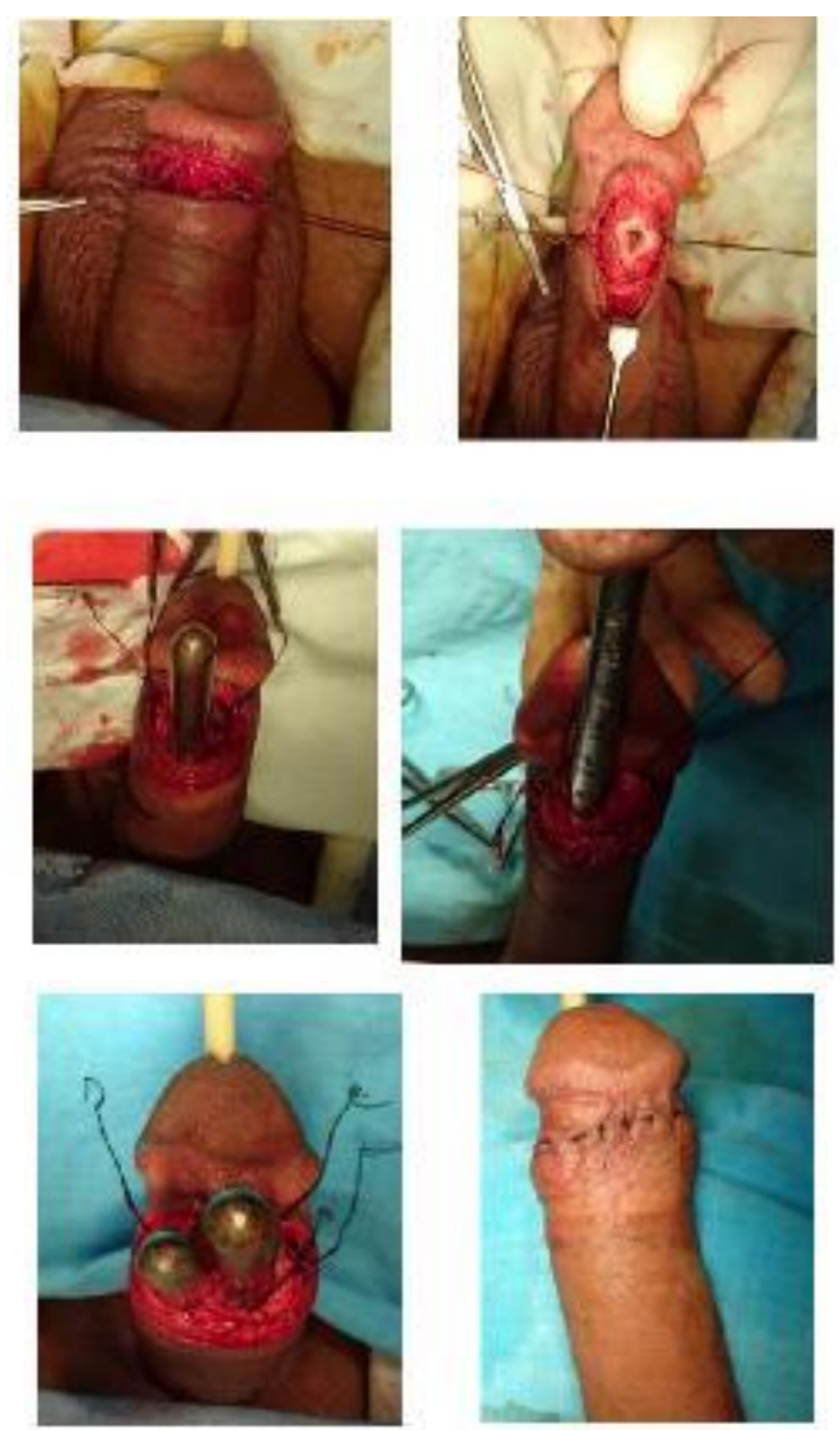

Figure 3: steps of penile prostheses implantation by subcoronal approach

\section{DISCUSSION}

The implantation of a penile prosthesis is still a gold standard therapy for men with ED who do not respond to pharmacologic agents. Patients often complain of penile length loss after prosthesis surgery. Despite this fact this treatment strategy had the highest reported satisfaction rates for patients and their partners ${ }^{(6)}$.The introduction of phosphodiesterase inhibitors had revolutionized the treatment of these patients, however, surgery remains clear because it represents the last treatment option when all others fail or were contraindicated ${ }^{(7)}$.Semi-rigid prostheses were the most suitable for elderly patients, with manual and mental limitations. Its mechanical reliability was excellent ${ }^{(4)}$. In our study, more than $40 \%$ above 50 years and dealing with the prostheses were more realizable. IIEF was widely used for the evaluation of male sexual activity because of its high degree of sensitivity and specificity. The evaluation was based on five main domains, namely erectile function, orgasmic function, sexual desire, satisfaction during intercourse and general satisfaction. Within erectile function, two items were specifically specified to assess ED, penetration capacity and ability to maintain sufficient erection for satisfactory sexual activity ${ }^{(8)}$. In all patients, careful sizing of the cylinders is critical for optimizing concealment and decreasing the incidence of dreaded complications such as erosion and chronic penile pain ${ }^{(9)}$. The critical point of surgery was cylinder size adequacy. If it was too large, it leads to persistent pain, protruding cylinders in the glands or penile curvature during 
erection ${ }^{(4)}$. In this study, we had one case that underwent revision due to oversizing. Semirigid prostheses were easier to handle and had lower rates of mechanical failure ${ }^{(4)}$. All patients in this study had no problem in dealing with the prostheses. Patient satisfaction was a complex issue that was related to many factors such as postoperative complications, cosmetic outcome, implant function and partner acceptability ${ }^{(10)}$. All the treatments available for ED, PPs had the highest rate of satisfaction for both patient and partner, although it is the least chosen method (4). All cases in this study were on medical treatment of ED for more than 1year and some of them had some trails in other methods of ED management, and the highest rate of satisfaction for both patient and partner was with penile prostheses. Eisenman reported that not the penile length but, the width was more important for women's sexual satisfaction $^{(11)}$. This study not meet with this report as penile length is one of important factor in satisfaction for both patient and his partner. It is important that the surgeon thoroughly discussed the potential limitations of prosthetic implantation and in particular, any unrealistic expectations regarding penile length. Penile prostheses do not increase the length of the penis. With this firmly in mind, patients with severe cavernosal fibrosis who had underwent prosthesis implantation had experience insufficient length that may be suitable candidates for penile lengthening with flap advancement ${ }^{(12)}$.

This study is in agreement with the previous study since we informed our patients about roll of implant in treatment of ED and no roll in increasing length of penis. Cylinder crossover can be difficult to recognize although it was believed to occur in up to $25 \%$ of cases ${ }^{(13)}$. This study showed that as device crossover had been occurred more with subcoronal approach this occurred when there was perforation of the intercavernous septum. This can be prevented by aiming the dilators laterally and dorsally. Crossover was easily corrected by placing a large dilator into the receiving side of the crossover and then redilating with subsequent cylinder placement on the contralateral side.

The use of semirigid devices in patients with Peyronie's disease is controversial. Adequate penile straightening was also reported by
Montorsi et al. but they observed diminishing patient satisfaction with time ${ }^{(9)}$.

This study meeting with that about penile straightening in patient with Peyronie's disease, but not meeting about patient satisfaction as patient and his partner are highly satisfied till the last visit after 1 year of operation.

Patients and their partners may become dissatisfied with a semirigid device for a variety of reasons, usually stemming from inability of the implants to adequately mimic a normal, physiologic erection. Reasons for dissatisfaction include chronic penile pain, numbness, diminished quality of orgasm, suboptimal penile length and/or girth, difficulty with concealment, and inadequate rigidity ${ }^{(9)}$. This study showed that most reason of dissatisfaction mentioned above, but not meeting about chronic penile pain and inadequate rigidity as all cases not remember these reasons in dissatisfaction. While, the semi-rigid prostheses can be implanted through a sub-coronal incision, currently the majority of prosthesis implanted in the USA was placed by using a penoscrotal approach. The penoscrotal incision offered excellent exposure of the corporal bodies and makes avoiding the dorsal sensory nerves easier ${ }^{(13)}$. In this study we preferred to use the penoscrotal approach during implantation of the semi-rigid prostheses for the reason mentioned above in addition to avoid glanular affection which result in some cases during using the subcoronal approach which affect patient satisfaction later on.

\section{Conclusion}

Although penile prostheses implantation are the most invasive treatment option, penile prostheses guarantee the highest satisfaction rate among all available ED treatment options and provide a predictable and reliable result; and the penoscrotal approach more suitable for semi-rigid penile prostheses than subcoronal one.

\section{REFERENCES}

1. Lue TF (2000): Erectile dysfunction. New Engl. J. Med., 342: 1802-1813.

2. Liu DF, Jiang H, Hong K, Zhao LM, Ma LL et al. (2009): Epidemiological changes of ED patients: investigations in 11 Chinese cities during the past 5 years]. Zhonghua Nan Ke Xue, 15: 724-730. 
3. Hellstrom WJ, Gittelman M, Karlin G, Segerson T, Thibonnier M et al. (2003): Sustained efficacy and tolerability of vardenafil, a highly potent selective phosphodiesterase type 5 inhibitor, in men with erectile dysfunction: results of a randomized, doubleblind, 26-week placebo-controlled pivotal trial. Urology, 61: 8-14.

4. Mulcahy JJ, Austoni E, Barada JH, Choi HK, Hellstrom WJ et al. (2004): The penile implant for erectile dysfunction. J. Sex. Med.,1: 98-109.

5. Glina $S$ and Martins F (2002): Técnicas de implante. In: Disfunção Sexual Masculina. São Paulo, Inst. H Hellis. Pp: 259-262.

6. Brinkman MJ, Henry GD, Wilson SK et al. (2005): A survey of patients with inflatable penile prosthesis for satisfaction. J. Urol., 174:253-260.

7. Grant P, Jackson G, Baig I and Quin J (2013): Erectile dysfunction in general medicine. Available on: http://www.ncbi.nlm.nih.gov/pubmed/236 81859.
8. Rosen RC, Cappelleri JC, Gendrano N (2002). The International Index of Erectile Function (IIEF): a state-of-the-science review.

http://www.ncbi.nlm.nih.gov/pubmed/121 52111

9. Brandell A and Thrasher J (2002): History of urologic prostheses. Urologic Prostheses, 10:170-178.

10. Mulhall JP, Absaar A, Branch $J$ and Parker M (2003): Serial assessment of efficacy and satisfaction profiles following penile prosthesis surgery. J. Urol., 169:1429-1433.

11. Eisenmen R (2001): Penis size: survey of female perceptions of sexual satisfaction. BMC Women's Health, 2001: 1:9.

12. Knoll LD, Fisher J, Benson RC, Minich PJ and FurlowWL (1996): Managing penile fibrosis with prosthetic implantation and flap advancement with tissue debulking. J. Urol., 156: 394-397.

13. Kenneth J, DeLay $J$ and Tobias $S$ (2016): Contemporary treatment of erectile dysfunction. Contemporary Endocrinology, DOI: 10.1007/978-3-31931587-4_15. 